\title{
Sutura laparoscópica frente a sutura por laparotomía en úlceras gastroduodenales perforadas
}

\author{
Patricia Ciriano H. ${ }^{1}$, Irene Grao T. ${ }^{1}$, Cristina Ruiz M. ${ }^{1}$, \\ Leticia Seisdedos R. ${ }^{1}$, Silvia Kayser M. ${ }^{1}$, Cristina Rey V. ${ }^{1}$, \\ María Dolores Pérez D. ${ }^{1}$ y Fernando Turegano F. ${ }^{1}$
}

${ }^{1}$ Hospital General Universitario Gregorio Marañón, Madrid, España.

Recibido el 7 de octubre de 2017, aceptado para publicación el 26 de octubre de 2017.

Correspondencia a: Patricia Ciriano $\mathrm{H}$. patricia.ciriano.hernandez@ gmail.com

\section{Laparoscopic suture facing laparotomy suture in perforated gastroduodenal ulcers}

Introduction: Gastroduodenal perforation continues to be a relatively frequent surgical emergency, despite advances in the medical treatment of ulcer disease. Its laparoscopic approach has been increasing in the last years, although it has not been generalized. Objective: Was to analyze the postoperative results in patients with perforated ulcer treated with laparoscopic suture, and to compare them with a similar group with laparotomy suture. Our hypothesis was that laparoscopic suture is a safe option and with less morbidity than the laparotomy approach. Material and Methods: Comparative retrospective analysis of two patient cohorts: one treated with laparoscopic suture during 2014 and 2015, a period in which this approach was fully implanted in the emergency room in our hospital, and another comparable group treated by suture for open surgery during the period 2001-2003. Complications were analyzed according to Clavien-Dindo classification, conversion rate, mean stay and mortality. Results: The groups were comparable in age, sex, comorbidities and anesthetic risk. There was a trend towards superiority in favor of the laparoscopic approach in certain variables analyzed, with a conversion rate of $3 \%$. The presence of early postoperative complications was greater in the laparotomy suture group: post-surgical septic shock $(15.2 \%$ vs $6 \%)$ and wound infection (15.2\% vs 3\%), as well as medical complications, although not significantly. The group with laparoscopic suture had a longer surgical time, lower mean stay and lower mortality. Conclusion: The laparoscopic suture of the gastroduodenal ulcer in our center has had a very low conversion rate and a somewhat lower morbidity to the laparotomy suture, with a lower rate of reinterventions and a mean stay, despite a longer surgical time.

Key words: laparoscopy, suture.

\section{Resumen}

Introducción: La perforación gastroduodenal continúa siendo una urgencia quirúrgica relativamente frecuente, a pesar de los avances realizados en el tratamiento médico de la enfermedad ulcerosa. Su abordaje laparoscópico ha ido aumentando en los últimos años, aunque no se ha generalizado. Nuestro objetivo es analizar los resultados postoperatorios en pacientes con úlcera perforada tratados mediante sutura laparoscópica, y compararlos con un grupo similar con sutura por laparotomía. Mantenemos la hipótesis de que la sutura laparoscópica es una opción segura y con menor morbilidad que el abordaje por laparotomía. Material y Métodos: Análisis retrospectivo comparativo de dos cohortes de pacientes: una tratada mediante sutura laparoscópica durante los años 2014 y 2015, período en el que este abordaje estaba plenamente implantado en la Urgencia en nuestro hospital, y otro grupo comparable tratado mediante sutura por cirugía abierta durante el período 2001-2003. Se analizaron las complicaciones según la clasificación de Clavien-Dindo, tasa de conversión, estancia media y mortalidad. Resultados: Los grupos eran comparables en edad, sexo, comorbilidades y riesgo anestésico. Se observó una tendencia a la superioridad a favor del abordaje laparoscópico en ciertas variables analizadas, con una tasa de conversión de un $3 \%$. La presencia de complicaciones postoperatorias precoces fue mayor en el grupo con sutura 
por laparotomía: shock séptico postquirúrgico ( $15,2 \%$ vs $6 \%)$ e infección de herida ( $15,2 \%$ vs 3\%), así como las complicaciones médicas, aunque de forma no significativa. El grupo tratado con sutura laparoscópica tuvo un mayor tiempo quirúrgico, menor estancia media y menor mortalidad. Conclusión: La sutura laparoscópica de la úlcera gastroduodenal en nuestro centro ha tenido una baja tasa de conversión y una morbilidad algo menor a la sutura por laparotomía, con una menor tasa de reintervenciones y menor estancia media, a pesar de un mayor tiempo quirúrgico.

Palabras clave: perforación, úlcera.

\section{Introducción}

Durante las últimas décadas el tratamiento de la úlcera gastroduodenal (UGD) ha cambiado radicalmente debido a la introducción de la triple terapia contra el Helicobacter pylori, identificado como causante de este tipo de patología ${ }^{1,3}$. Esto hace que en la actualidad apenas se practiquen técnicas encaminadas a controlar la secreción ácida gástrica, pero llama la atención que la frecuencia de complicaciones se mantiene estable a pesar de estos avances experimentados en el tratamiento médico.

El riesgo de presentar complicaciones es del 3 al $6 \%$ de las UGD, pudiendo llegar hasta el 10\% dependiendo de las fuentes bibliográficas consultadas. Dentro de las complicaciones, las más frecuentes continúan siendo la hemorragia y la perforación, entendida esta última como la disrupción completa de todas las capas de la pared, lo que permite el vertido de gas, ácido gástrico y líquido biliar al interior de la cavidad abdominal. Este vertido genera una reacción peritoneal que puede ser generalizada o localizada, si la perforación está contenida ${ }^{2-5}$.

El tratamiento de la perforación continúa siendo mayoritariamente quirúrgico, siendo de elección en nuestro centro la sutura, con o sin omentoplastía añadida. Otros prefieren simplemente una omentoplastía, a modo de parche que cubre la perforación ${ }^{2,3,6}$.

El abordaje laparoscópico de la UGD perforada se describió por primera vez en 1989, tras la utilización de pegamento de fibrina y parche omental por Mouret y cols ${ }^{7}$. Posteriormente, en 1990, Nathanson y cols refieren la primera sutura y omentoplastía ${ }^{8}$. La sencillez de la técnica por vía laparoscópica, y sus ventajas potenciales respecto a la sutura por laparotomía, ha hecho que en muchos grupos como el nuestro constituya la técnica de elección en los últimos años; sin embargo, dificultades en el uso de la laparoscopia en Urgencias, el deseo de mantener unos tiempos quirúrgicos cortos, o incluso dudas sobre la seguridad y efectividad del cierre laparoscópico hace que en muchos medios se siga prefiriendo el cierre por laparotomía. Otros reservan la laparoscopia para pacientes con mejor estado fisiológico, empleando la laparotomía, en general por el menor tiempo quirúrgico, en aquellos pacientes más comprometidos ${ }^{3,9}$.

Nuestro objetivo fue evaluar los resultados de ambos abordajes quirúrgicos, con la hipótesis de que la sutura por laparoscopia se compara favorablemente con la realizada por laparotomía y supondría un indudable avance terapéutico.

\section{Material y Métodos}

Se trata de un estudio retrospectivo comparando dos cohortes de pacientes intervenidos de forma consecutiva por perforación de UGD: un grupo de abordaje laparoscópico (cirugía laparoscópica-CLP) durante los años 2014 y 2015, y un grupo comparable intervenido por laparotomía (cirugía abierta-CA) durante los años 2001-2003, época en que la técnica laparoscópica aún no se había incorporado al tratamiento de esta patología urgente en nuestro centro.

Se analizaron las características demográficas de los pacientes, comorbilidades, antecedentes de cirugía abdominal, diagnóstico previo de enfermedad ulcerosa, toma previa de AINEs, técnica y tiempo quirúrgico, morbilidad a corto y medio plazo (eventración o hernia del trocar, con un tiempo de seguimiento máximo de 2 años), y la estancia hospitalaria. Se comprobó que los grupos fueran comparables en características demográficas, comorbilidades y ASA, para minimizar al máximo posible el sesgo de selección de pacientes, que desviaría a aquellos con indicadores de mayor gravedad al grupo de cirugía abierta, por el menor tiempo quirúrgico.

El análisis estadístico se realizó utilizando el programa IBM SPSS Statistics versión $n^{\circ} 19$. Las dos cohortes se compararon aplicando los test chicuadrado y el test exacto de Fisher para el análisis de variables cualitativas. Para la comparación de variables cuantitativas se utilizaron pruebas no paramétricas (U de Mann Whitney). Hemos consi- 
derado como estadísticamente significativo un valor $\mathrm{p}<0,05$.

Se analizó el lugar de perforación (gástrica, prepilórica o duodenal), y la necesidad de conversión a cirugía abierta. En ambos grupos la técnica empleada fue la sutura simple de la perforación, con o sin omentoplastía asociada. Se excluyeron, por tanto, del estudio aquellos pacientes tratados mediante vagotomía + piloroplastía y/o resección gástrica total o subtotal.

Se analizaron las complicaciones postoperatorias según la clasificación de Clavien-Dindo ${ }^{10,11}$ que define el tipo de complicación en base al tratamiento que precisa, siendo la principal limitación la dificultad para registrar aquellas complicaciones más leves (Grado I) que no precisan de tratamiento médico ni quirúrgico de ningún tipo. Se consideraron complicaciones Grado II aquellas tratadas farmacológicamente como las infecciones respiratorias y patología cardiológica, en su mayor parte alteraciones del ritmo cardiaco. Complicaciones Grado III, las infecciones de herida, fugas y colecciones intraabdominales que precisaron drenaje percutáneo mediante radiología intervencionista (Grado IIIA) o reintervención (Grado IIIB). Finalmente se consideraron como Grado IV el estado de disfunción uni o multiorgánica (Tipo IV A y B, respectivamente) que precisaron ingreso en UCI. La mortalidad precoz o complicación Grado V se definió como aquella que se produjo durante los primeros 30 días postoperatorios.

\section{Resultados}

De los 66 pacientes analizados, 33 pertenecen al grupo de CA y los 33 restantes al de CLP. Al ser un análisis comparativo de pacientes tratados de manera consecutiva en el tiempo, y centrar nuestro análisis en la técnica quirúrgica más sencilla (sutura \pm omentoplastía), se excluyeron 15 pacientes tratados con vagotomía y piloroplastía (2 en el grupo CLP y 13 en el grupo CA), y 5 pacientes tratados con resección gástrica en el grupo $\mathrm{CA}$ durante los períodos de tiempo señalados.

Los dos grupos eran comparables en las variables demográficas analizadas, así como en antecedentes y clasificación ASA (Tabla 1 ). Un $12 \%$ de pacientes en el grupo CA presentaron antecedentes de úlcera gastroduodenal, frente a un $6 \%$ de pacientes en el caso de CLP.

La Tabla 2 muestra la localización de la perforación y la técnica quirúrgica empleada, no existiendo diferencias entre los dos abordajes.
La Tabla 3 muestra que las complicaciones médicas (Grado II de Clavien-Dindo) fueron más frecuentes en el grupo CA, aunque sin alcanzar significación estadística probablemente por el pequeño tamaño muestral. La tasa de infección de herida quirúrgica, Grado III de Clavien- Dindo, fue también mayor en el grupo CA. La tasa de colecciones intraabdominales fue pequeña, no existiendo diferencias entre los dos grupos, siendo tratadas mediante drenaje percutáneo. Las 3 fugas de sutura en el grupo CLP requirieron laparotomía urgente (Grado IIIB de Clavien-Dindo) y nueva sutura en dos casos, y una gastrectomía subtotal en el otro. En los dos casos del grupo CA se hizo gastrectomía subtotal. La presencia de sepsis postquirúrgica asociada a fracaso uni o multiorgánico (Grado IV de la clasificación Clavien-Dindo) fue mayor en CA, aunque sin diferencias estadísticamente significativas.

Tabla 1. Características demográficas, antecedentes y clasificación ASA

\begin{tabular}{|lccc|}
\hline & $\begin{array}{c}\text { Grupo CLP } \\
(\mathbf{n = 3 3 )}\end{array}$ & $\begin{array}{c}\text { Grupo CA } \\
(\mathbf{n = 3 3 )}\end{array}$ & p \\
Edad & $51(17-94)$ & $56(22-94)$ & n.s. \\
Sexo hombre & $23(70 \%)$ & $20(60 \%)$ & n.s. \\
Sexo mujer & $10(30 \%)$ & $13(40 \%)$ & \\
Cirugía previa & $10(30 \%)$ & $9(27,3 \%)$ & n.s. \\
Comorbilidad & $10(30 \%)$ & $15(45,5 \%)$ & 0,21 \\
ASA I & $15(45,5 \%)$ & $16(48,5 \%)$ & \\
ASA II & $15(45,5 \%)$ & $14(42,5 \%)$ & 0,73 \\
ASA III & $2(6 \%)$ & $3(9 \%)$ & \\
ASA IV & $1(3 \%)$ & $0(0 \%)$ & \\
\hline
\end{tabular}

ASA: American Society of Anesthesiology. CLP: Cirugía laparoscópica. CA: Cirugía abierta.

Tabla 2. Localización de la perforación y técnica quirúrgica

\begin{tabular}{|lccc|}
\hline & $\begin{array}{c}\text { Grupo CLP } \\
(\mathbf{n = 3 3 )}\end{array}$ & $\begin{array}{c}\text { Grupo CA } \\
(\mathbf{n = 3 3 )}\end{array}$ & p \\
Gástrica & $6(18 \%)$ & $5(15 \%)$ & \\
Prepilórica & $14(42,5 \%)$ & $21(64 \%)$ & 0,19 \\
Duodenal & $13(39,5 \%)$ & $7(21 \%)$ & \\
Sutura & $10(30 \%)$ & $16(48,5 \%)$ & \\
$\begin{array}{l}\text { Sutura }+ \\
\text { omentoplastía }\end{array}$ & $23(70 \%)$ & $17(51,5 \%)$ & 0,13 \\
\hline
\end{tabular}


ARTÍCULO ORIGINAL

La Tabla 4 muestra la ausencia de diferencias significativas en cuanto a necesidad de reintervención, mortalidad y desarrollo de hernia de trocar o eventración, aunque sí las hubo, de manera significativa, en el tiempo quirúrgico y estancia media hospitalaria. En tres de los 5 casos reintervenidos del grupo CA la causa fue una evisceración. Hubo un $12 \%$ de pérdidas en el seguimiento en el grupo CLP frente al $27 \%$ en el CA, probablemente debido a la mejor recogida de datos de los pacientes durante los últimos años tras la implantación de la historia clínica electrónica.

\section{Discusión}

A pesar de las mejoras experimentadas por el tratamiento médico durante las últimas décadas, las complicaciones de la patología ulcerosa como la hemorragia y la perforación continúan siendo relativamente frecuentes, siendo la cirugía urgente necesaria en una mayoría de perforaciones ${ }^{1-4}$.

El abordaje laparoscópico de la perforación gastroduodenal se describió hace aproximadamente dos, décadas ${ }^{7,8}$ y su uso ha ido creciendo, aunque no se ha generalizado. El mayor tiempo quirúrgico empleado, la supuesta menor fiabilidad de la sutura por vía laparoscópica en tejidos inflamados, y la teórica mayor dificultad en la limpieza de la cavidad abdominal en peritonitis establecida, con mayores colecciones residuales potenciales, han sido citados como argumentos en su contra ${ }^{3,9}$. Además, en presencia de adherencias por laparotomías previas podría existir una mayor dificultad en la exploración laparoscópica, algo que desmiente nuestro 30\% de pacientes abordados por laparoscopia que tenían cirugía abdominal.

Hoy en día la única contraindicación absoluta al abordaje laparoscópico podría ser la inestabilidad hemodinámica ya que, en general, los pacientes de más riesgo por comorbilidades y más sépticos podrían incluso beneficiarse de una laparoscopia a baja presión, lo que haría posible realizar la técnica de forma satisfactoria y con las ventajas de este tipo de abordaje en cuanto a recuperación precoz, menor requerimiento analgésico y reincorporación temprana a su actividad habitual ${ }^{5}$.

El avance en el tratamiento médico ha hecho cambiar la técnica quirúrgica, pasando de una mayoría de pacientes tratados mediante vagotomía y piloroplastía en el pasado, al cierre con parche de epiplón, o la sutura simple de la perforación, reforzada o no con parche omental ${ }^{5}$. Las intervenciones más complejas, encaminadas a modificar la
Tabla 3. Complicaciones postquirúrgicas según la clasificación de Clavien-Dindo

\begin{tabular}{|lccc|}
\hline & $\begin{array}{c}\text { Grupo CLP } \\
(\mathbf{n}=\mathbf{3 3})\end{array}$ & $\begin{array}{c}\text { Grupo CA } \\
(\mathbf{n}=\mathbf{3 3})\end{array}$ & $\mathbf{p}$ \\
\hline Respiratoria (II) & $2(6 \%)$ & $6(18 \%)$ & 0,13 \\
Cardiológica (II) & $0(0 \%)$ & $3(9 \%)$ & 0,07 \\
Infección herida (III) & $1(3 \%)$ & $5(15,2 \%)$ & 0,12 \\
Colección intraabdominal (IIIA) & $2(6 \%)$ & $3(9 \%)$ & 0,64 \\
Fuga de la sutura (IIIB) & $3(9 \%)$ & $2(6 \%)$ & 0,55 \\
Shock séptico (IV A y B) & $2(6 \%)$ & $5(15,2 \%)$ & 0,23 \\
\hline
\end{tabular}

Tabla 4. Resultados postquirúrgicos

\begin{tabular}{|lccc|}
\hline & $\begin{array}{c}\text { Grupo CLP } \\
(\mathbf{n = 3 3 )}\end{array}$ & $\begin{array}{c}\text { Grupo CA } \\
(\mathbf{n}=\mathbf{3 3})\end{array}$ & p \\
\hline Mortalidad (30 días) & $1(3 \%)$ & $2(6 \%)$ & 0,63 \\
Eventración (a 2 años) & $2(6 \%)$ & $3(9 \%)$ & 0,54 \\
Reintervención & $3(9 \%)$ & $5(15,2 \%)$ & 0,45 \\
Conversión & $1(3 \%)$ & N.A. & \\
Tiempo quirúrgico en min. & $106(40-170)$ & $67(35-255)$ & 0,001 \\
EM hospitalaria en días & $8(3-19)$ & $15(1-91)$ & 0,001 \\
\hline
\end{tabular}

N.A: No Aplicable; EM: Estancia Media.

secreción ácida, se reservan a los escasos pacientes en que se sospeche que no se va a realizar un tratamiento médico posterior fiable ${ }^{3,4}$.

De acuerdo con diferentes estudios a favor del tratamiento laparoscópico podríamos destacar una menor frecuencia de infección del sitio quirúrgico y de sepsis posoperatoria. En nuestro estudio también hemos observado una disminución de complicaciones médicas, fundamentalmente respiratorias y cardiológicas, quizás debido a un menor dolor posoperatorio que favorece la fisioterapia respiratoria, siendo estos resultados similares a los de otros grupos $^{2,3,5,6}$.

En contraste con otros estudios, con cifras que oscilan entre el $12 \%^{2}$ y el $24 \%{ }^{6}$, nuestra tasa de conversión $(3 \%)$ resultó anecdótica. En cuanto a la de reintervención, fue algo menor en el grupo CLP (9\% vs 15,2\%), y en cualquier caso menor al $17 \%$ de un estudio de referencia ${ }^{6}$. Ambos resultados fueron, sin embargo, desfavorables en comparación con los obtenidos por Byrge y cols ${ }^{5}$, en el que las tasas de reintervención fueron del $4 \%$ y del $8 \%$, siendo favorable al abordaje laparoscópico, aunque no de forma estadísticamente significativa $(p=0,7)$. 
En cuanto a las causas, todos los estudios citados están de acuerdo con el nuestro en que, en los casos tratados mediante laparoscopia, se deben, principalmente, a fugas en la sutura ${ }^{3,6}$. En nuestra serie, la evisceración postlaparotomía fue la principal causa de reintervención (3 pacientes), seguido por la fuga de sutura (2 pacientes).

El principal inconveniente del abordaje laparoscópico en nuestra serie fue el aumento del tiempo quirúrgico, a diferencia de grupos como el de Kirshtein y $\operatorname{cols}^{2}$, o el de Matthias y cols ${ }^{3}$, en el que no hubo diferencias estadísticamente significativas en cuanto al tiempo quirúrgico. En nuestro centro esto quedaría justificado por la introducción relativamente reciente del abordaje laparoscópico en la cirugía de urgencia y la necesaria curva de aprendizaje.

La reducción en la estancia media en el grupo CLP frente al grupo CA fue acorde con la obtenida por otros grupos como el de Byrge y $\operatorname{col}^{5}$, aunque en otro estudio ${ }^{3}$ no se apreció diferencia estadísticamente significativa.

Como principal limitación de nuestro estudio cabe destacar la pérdida de seguimiento de algunos pacientes, lo que favorece el subdiagnóstico de la hernia incisional a largo plazo. Por otra parte, creemos que el tamaño muestral relativamente pequeño favoreció que la mayoría de resultados no fueran estadísticamente significativos, a pesar de quedar patente una tendencia hacia la superioridad del abordaje laparoscópico en cuanto a la disminución de morbilidad y estancia media.

En conclusión, la sutura laparoscópica de la perforación gastroduodenal en nuestro centro ha tenido una baja tasa de conversión y una morbilidad com- parable a la sutura por laparotomía, con una menor tasa de reintervenciones y menor estancia media, a pesar de un mayor tiempo quirúrgico.

\section{Responsabilidades éticas}

Protección de personas y animales. Los autores declaran que para esta investigación no se han realizado experimentos en seres humanos ni en animales.

Confidencialidad de los datos. Los autores declaran que han seguido los protocolos de su centro de trabajo sobre la publicación de datos de pacientes.

Derecho a la privacidad y consentimiento informado. Los autores declaran que en este artículo no aparecen datos de pacientes.

\section{Financiación}

Los autores declaran no haber recibido ninguna financiación para la realización de este trabajo.

\section{Conflicto de intereses}

Los autores declaran no tener ningún conflicto de intereses.

\section{Agradecimientos}

Gracias al hospital universitario Gregorio Marañón por proporcionar los datos necesarios para la realización del estudio y a los doctores Turégano, Pérez Díaz y Rey por la colaboración en la realización del estudio.

\section{Bibliografía}

1. Agrusa A, Romano G, Di Buono G, Dafnomili A, Gulotta G. Laparoscopic approach in abdominal emergencies: a 5 -year experience at a single center. C Chir. 2012; 33:400-3.

2. Kirshtein B, Bayme M, Mayer T, Lantsberg L, Avinoach E, Mizrahi S. Laparoscopic treatment of gastroduodenal perforations: Comparison with conventional surgery. Surg Endosc. 2005;19:1487-90.

3. Matthias MH, Seelig SK, Behn C, Schonleben K. Comparison Between Open and Laparoscopic Technique in the Management of Perforated Gastroduodenal Ulcers. J Clin Gastroenterol. 2003;37:226-9.
4. Nirula R. Gastroduodenal Perforation. Surg Clin N Am. 2014;94:31-4.

5. Byrge N, Barton RG, Enniss TM, Nirula R. Laparoscopic versus open repair of perforated gastroduodenal ulcer: a National Surgical Quality Improvement Program analysis. Am J Surg. 2013;206:957-63.

6. Wilhelmsen M, Møllerand MH, Rosenstock S. Surgical complications after open and laparoscopic surgery for perforated peptic ulcer in a nationwide cohort. BJS 2015;102: 382-7.

7. Mouret P, François Y, Vignal J, Barth $\mathrm{X}$, Lombard- Platet R. Laparoscopic treatment of perforated peptic ulcer. Br J Surg. 1990;77:1006

8. Nathanson LK, Easter DW, Cuschieri A
Laparoscopic repair/peritoneal toilet of perforated duodenal ulcer. Surg Endosc. 1990;4:232-3.

9. Soreide $\mathrm{K}$, Thorsen $\mathrm{K}$, Harrison EM, Bingener J, Moller MH, Ohene Yeboah $\mathrm{M}$, et al. Perforated peptic ulcer. Lancet. 2015;26;386:1288-98.

10. Dindo C, Demartines M, Clavien PA. Classification of surgical complications. A new proposal with evaluation in a cohort of 6336 patients and results of survey. Ann Surg. 2004;240:205-13.

11. Katayama H, Kurokawa Y, Nakamura $\mathrm{K}$, Ito H, Kanemitsu Y, Masuda N, et al. Extended Clavien-Dindo classification of surgical complications: Japan Clinic Oncology Group postoperative complications criteria. Surg Today 2016;46:668- 85 . 